\title{
High yield production of L-serine through a novel identified exporter combined with synthetic pathway in Corynebacterium glutamicum
}

\section{Xiaomei Zhang}

Jiangnan University

\section{Yujie Gao}

Jiangnan University

\section{Ziwei Chen}

Jiangnan University

\section{Guoqiang Xu}

Jiangnan University

Xiaojuan Zhang

Jiangnan University

Hui Li

Jiangnan University

Jinsong Shi

Jiangnan University

Mattheos Koffas ( $\square$ koffam@rpi.edu )

Rensselaer Polytechnic Institute https://orcid.org/0000-0002-1405-0565

Zhenghong Xu ( $\sim$ zhenghxu@jiangnan.edu.cn)

Jiangnan University

\section{Research}

Keywords: L-serine, exporter, C. glutamicum, transcriptional regulator, metabolic engineering

Posted Date: March 12th, 2020

DOI: https://doi.org/10.21203/rs.3.rs-16998/v1

License: (c) (i) This work is licensed under a Creative Commons Attribution 4.0 International License.

Read Full License 


\section{Abstract}

Background L-Serine has wide and expanding applications in industry with a fast-growing market demand. Strategies for achieving and improving L-serine production in C. glutamicum have focused on inhibiting its degradation and enhancing its biosynthetic pathway, however, L-serine yield remains relatively low. Exporters play an essential role in the fermentative production of amino acids. Improvements to L-serine yield should consider the improvement of L-serine export from the cell. In C. glutamicum , ThrE, which can export L-threonine and L-serine, is the only identified L-serine exporter so far.

Results In this study, a novel L-serine exporter NCgl0580 was identified and characterized in C. glutamicum $\triangle$ SSAAI (SSAAI), and named as SerE (encoded by serE ). Deletion of serE in SSAAl led to a $56.5 \%$ decrease in L-serine titer, whereas overexpression of serE compensated for the lack of serE with respect to L-serine titer. A fusion protein with SerE and enhanced green fluorescent protein (EGFP) was constructed to confirm that SerE localized at the plasma membrane. The function of SerE was studied by peptide feeding approaches, and the results showed that SerE is a novel exporter for L-serine and Lthreonine in C. glutamicum. Subsequently, the interaction of a known L-serine exporter ThrE and SerE was studied, and the results suggested that SerE is more important than ThrE in L-serine export in SSAAI. In addition, probe plasmid and electrophoretic mobility shift assays (EMSA) revealed NCgI0581 as the transcriptional regulator of SerE. Comparative transcriptomics between SSAAI and the NCgI0581 deletion strain showed that $\mathrm{NCgl0581}$ is a positive regulator of NCgl0580. Finally, by overexpressing the novel exporter SerE combined with L-serine synthetic pathway key enzyme serA $\Delta 197$, serC and serB, the resulting strain lead to the $\mathrm{L}$-serine titer of $43.9 \mathrm{~g} / \mathrm{L}$ with yield of $0.44 \mathrm{~g} / \mathrm{g}$ sucrose, which was the highest yield reported so far for any organism.

Conclusions This study provides a novel target for L-serine and L-threonine export engineering as well as a novel global transcriptional regulator NCgl0581 in C. glutamicum .

\section{Background}

L-serine has been identified as one of the top 30 most interesting building blocks for a range of chemicals and materials, and is used in cosmetic, pharmaceutical, and food industries [1, 2]. Metabolic engineering of Corynebacterium glutamicum (C.glutamicum) for L-serine production has been focused on its terminal synthesis pathways and degradation pathways, and proven to be very useful for improving L-serine production in this organism [3-6]; however, the L-serine productivity is still low for large-scale L-serine production, the highest reported L-serine titer reached $42.62 \mathrm{~g} / \mathrm{L}$ and $50 \mathrm{~g} / \mathrm{L}$ with the yield to be $0.21 \mathrm{~g} / \mathrm{g}$ sucrose and $0.26 \mathrm{~g} / \mathrm{g}$ glucose in C. glutamicum and E.coli respectively, however, L-serine has the potential to be made from sugar by fermentation with a very high theoretical yield $(1.17 \mathrm{~g} / \mathrm{g}$ glucose, $1.22 \mathrm{~g} / \mathrm{g}$ sucrose) [2]. 
Improvements to L-serine production should consider the improvement of L-serine export from the cell. Export plays an essential role in metabolic engineering strategies for production of amino acids [7], as it reduces intracellular amino acid concentrations, and thereby alleviates feedback inhibition and circumvents toxicity problems [1, 8-10]. In recent decades, a number of export systems have been identified for excreting amino acids, such as L-lysine, L-cysteine, L-glutamate, L-threonine, L-arginine, Lmethionine, and branched-chain amino acids, in C. glutamicum and E.coli [11-17]. However, to the best of our knowledge, except for ThrE (L-threonine and L-serine exporter) $[15,18]$, no other L-serine exporters have been reported in C. glutamicum so far. In E. coli, Mundhada et al. found that intracellular L-serine accumulation was toxic to the engineered strain modified to produce L-serine, and that following overexpression of eamA (which encodes L-cysteine exporter in E. coli), the engineered strain exhibited increased tolerance toward L-serine with higher L-serine productivity [2]. Therefore, L-serine exporter in C. glutamicum could be a potential target for strain optimization to further improve L-serine production.

It has been reported that homologs similar to the exporters in E. coli might fulfil a comparable function in C. glutamicum $[17,19,20]$. Accordingly, we hypothesized that the homolog to eamA (L-serine exporter in E. coli) might be involved in L-serine export in C. glutamicum. In the present study, three homologs to eamA, namely, NCgl2050, NCgl2065, and NCgl0580, were determined, and their functions were identified by targeted gene deletion, respectively. The results showed that one of the genes, NCgl0580, was involved in L-serine export. Subsequently, localization and function of NCgl0580 were investigated, and the interaction of a known L-serine exporter ThrE (encoded by thrE) and the novel exporter NCgl0580 was studied. Furthermore, the transcriptional regulator of NCgl0580 was identified and studied. Finally, the effect of overexpression of L-serine exporter in combination with L-serine synthetic pathway enzyme to Lserine production were evaluated.

\section{Results}

\section{Exploring putative L-serine exporters in C. glutamicum}

In past studies, homologs of $E$. coli exporters have been shown to have similar functions in $C$. glutamicum $[17,19,20]$. Therefore, we hypothesized that the $C$. glutamicum homolog to eamA (L-serine exporter in E. coli) [2] might be involved in L-serine export in this organism. According to the NCBI database, EamA belongs to the RhaT superfamily, and 15 records of related proteins associated with RhaT superfamily in C. glutamicum ATCC13032 were obtained. After eliminating duplicate records, three related genes, NCgl2050, NCgI2065, and NCgl0580, were obtained, which might be involved in L-serine export in C. glutamicum.

To verify the function of these putative proteins in C. glutamicum SSAAI (SSAAI), NCgl2050, NCgl2065, and NCgl0580 were respectively deleted in this strain. The results showed that the deletion of NCgl2050 and NCgl2065 did not produce any changes in cell growth and L-serine titer (Fig. 1A and 1B). Strikingly, deletion of NCgl0580 significantly reduced the L-serine titer in SSAAl, but did not affect the growth of the strain (Fig. 1C). SSAAI $\triangle$ NCgl0580 produced $11.31 \mathrm{~g} / \mathrm{L}$ L-serine, which was $56.5 \%$ lower than that noted 
in SSAAI (Fig. 1C). However, plasmid-borne overexpression of NCgl0580 compensated for the lack of NCgl0580 with respect to L-serine titer, resulting in $26.76 \mathrm{~g} / \mathrm{L} \mathrm{L}$-serine titer, similar to that generated by the parent strain SSAAI (Fig. 1D). As shown in Fig. 1D, when compared with SSAAl, the strain harboring the plasmid grew slowly to some extent in the logarithmic growth phase, finally reaching similar cell growth to SSAAI. This finding suggested that NCgl0580 might act as the L-serine exporter in C. glutamicum, and was named as SerE and its function was further investigated.

\section{Localization and function of SerE}

According to the NCBI, SerE was presumed to be a hypothetical membrane protein of 301 amino acids, similar to permease of the drug/metabolite transporter (DMT) superfamily. The transmembrane helices of SerE were predicted by TMHMM Server v. 2.0, and SerE exhibited ten transmembrane-spanning helices with both amino- and carboxy-terminal ends in the cytoplasm.

To confirm the localization of SerE, SerE-EGFP fusion protein was expressed in SSAAI. Confocal microscopic observations of SSAAI-egfp and SSAAI-serE-egfp confirmed that EGFP and SerE-EGFP fusion proteins were successfully expressed, respectively (Fig. S1). To further verify the localization of SerE, membrane and cytoplasmic proteins from these two strains were extracted by ultrasonication, and the fluorescence of these proteins was determined using a fluorescence spectrophotometer. The fluorescence of the cytoplasmic proteins of SSAAI-egfp and membrane proteins of SSAAI-serE-egfp (Fig. 2A) affirmed that SerE was localized at the plasma membrane in SSAAI.

To substantiate the function of SerE, a peptide feeding approach was employed by incubating SSAAI and SerE deletion strain, SSAAI $\triangle$ serE, with $2 \mathrm{mM}$ of the dipeptide Ser-Ser, respectively, and measuring the concentration of extracellular L-serine. As shown in Fig. 2B, a higher L-serine concentration was detected in SSAAl, when compared with that in SSAAI $\triangle$ serE, confirming that SerE is a novel exporter of L-serine in C. glutamicum.

It is known that L-cysteine accept system in E. coli (encoded by eamA) also catalyzes L-serine accept [2], and that L-threonine exporter in C. glutamicum (encoded by thrE) also transports L-serine [15]. We therefore analyzed whether the novel exporter SerE could accept L-cysteine and L-threonine. The export experiments with dipeptides (Thr-Thr, Cys-Cys) were performed using SSAAI and SSAAI $\Delta$ serE. The dipeptides were added at a concentration of $2 \mathrm{mM}$ to the medium, and the extracellular amino acid concentrations at different time intervals were determined by HPLC. The results revealed that the concentration of L-cysteine was comparable in both strains and did not significantly change (data not shown), indicating that SerE does not export L-cysteine. Interestingly, the concentrations of L-threonine in SSAAI $\triangle$ serE were lower than those in SSAAI (Fig.2C), indicating that SerE is also an exporter of Lthreonine in C. glutamicum.

\section{Interaction of a known exporter ThrE and a novel exporter SerE}


It is well known that thrE encodes ThrE that can export L-threonine and L-serine in C. glutamicum ATCC13032 [15]. To understand the interaction of ThrE and SerE on L-serine accept, thrE was deleted in SSAAI (SSAAI $\triangle t h r E$ ), and no significant change was observed in L-serine excretion for the deletion mutant (Fig. 3A and 3B). In contrast, deletion of SerE significantly reduced the L-serine titer in SSAAI, and resulted in little change in cell growth (Fig. 1C). The SSAAI $\triangle$ serE produced $11.31 \mathrm{~g} / \mathrm{L} \mathrm{L-serine,} \mathrm{which} \mathrm{was}$ $56.5 \%$ lower than that produced by SSAAI (Fig. 1C). Subsequently, thrE and serE double deletion mutant was constructed, which exhibited cell growth comparable to that of SSAAI (Fig. 3B), and produced 10.34 g/L L-serine, which was $60 \%$ lower than that observed in SSAAI (Fig. 3A and. 3B).

Furthermore, thrE and serE were overexpressed alone or in combination in SSAAI, then SSAAI-thrE, SSAAIserE and SSAAl-thrE-serE were obtained. While L-serine accumulation in SSAAl-thrE was similar to that in SSAAI (Fig. 3A and 3C), the production of L-serine in SSAAl-serE reached $28.67 \mathrm{~g} / \mathrm{L}$, which was $10.5 \%$ higher than that noted in SSAAI (Fig. 3A and 3C). However, a decrease in cell growth was observed in SSAAl-serE before $72 \mathrm{~h}$ of fermentation, when compared with that found in SSAAI (Fig. 3C). And no significant difference of L-serine titer was found in the time courses of both SSAAI-serE and SSAAl-thrEserE (Fig. 3C), and SSAAl-thrE-serE exhibited a lower cell growth than SSAAl-thrE before $96 \mathrm{~h}$ of fermentation. These observations might be due to the fact that over-efflux L-serine inhibited the cell growth. And the results suggested that SerE is more important than ThrE in L-serine export in SSAAl.

\section{Transcriptional regulator of the novel exporter SerE}

The gene NCgI0581, located upstream of serE and divergently transcribed from serE (Fig. S2), and its product (consisting of 303 amino acids) was found to be a member of the LysR-type transcriptional regulators (LTTRs) family. It must be noted that LTTRs were initially described as regulators of divergently transcribed genes [21]. In a previous study on C. glutamicum, LysG, located upstream of L-lysine exporter gene lysE, was observed to encode a LysR-type transcriptional regulator, confirming that LysG is a positive transcriptional regulator of lysE [22]. Accordingly, we speculated that NCgl0581 might be involved in the control of serE transcription.

To determine the function of NCgl0581, a mutant strain with NCgl0581 deletion was constructed. As shown in Fig. 4A, the growth of SSAAI $\triangle \mathrm{NCgl0581}$ was similar to that of the parent strain SSAAl. However, the L-serine titer of SSAAI $\triangle$ NCgl0581 was $11.08 \mathrm{~g} / \mathrm{L}$, which was $57.4 \%$ lower than that of the parent strain, indicating that $\mathrm{NCgl} 0581$ played an important role in L-serine production. Subsequently, the effect of NCgl0581 on serE expression was further investigated by using the probe plasmid pDXW-11. Two recombinant strains, SSAAI $\triangle$ NCgl0581-1 (harboring the plasmid pDXW-11-1, Fig. S3A) and SSAAI $\triangle N C g 10581-0$ (harboring the plasmid pDXW-11-0, Fig. S3B) were constructed, and their fluorescence during fermentation was measured. The fluorescence of SSAAI $\triangle \mathrm{NCgl0581-1}$ was stronger than that of SSAAI $\triangle$ NCgI0581-0 during the fermentation process (Fig. S3C), revealing that NCgl0581 functioned as a

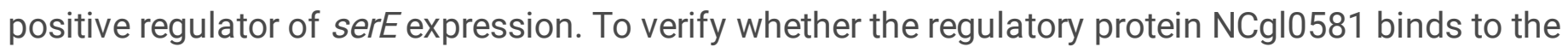
upstream region of SerE, electrophoretic mobility shift assay (EMSA) was performed by using the DNA probe labeled with biotin, and the result clearly indicated that NCgl0581 binds to this region (Fig. S4). 
To confirm whether NCgI0581 is a specific regulator of SerE, transcriptome sequencing was performed using SSAAI and NCgl0581 deletion strain. The findings showed that the transcription levels of 115 genes were altered, including 56 genes upregulated and 59 genes downregulated, in response to NCgl0581 deletion, indicating that NCgl0581 is a global transcriptional regulator in C. glutamicum. The genes with significant transcriptional change ( $\geq 4$-fold) are shown in Tables 1 and 2 .

TABLE 1 The genes significantly up regulated by NCgl0581 deletion

\begin{tabular}{|c|c|c|c|c|}
\hline Gene id & SSAAI $\Delta 0581$ & SSAAI & Fold change & Protein function \\
\hline NCgl2897 & 701.56 & 71.07 & 9.87 & Starvation-inducible DNA-binding protein \\
\hline NCgl0546 & 17.78 & 2.75 & 6.45 & Hypothetical protein \\
\hline NCgl1405 & 15.94 & 2.71 & 5.88 & ABC transporter periplasmic component \\
\hline NCgl1302 & 10.05 & 1.96 & 5.13 & Aldo/keto reductase \\
\hline NCgl1344 & 286.87 & 55.96 & 5.12 & Ornithine carbamoyltransferase \\
\hline NCgl1343 & 280.65 & 57.24 & 4.9 & Acetylornithine aminotransferase \\
\hline NCgl0746 & 43.30 & 9.04 & 4.7 & Hypothetical protein \\
\hline NCgl1342 & 134.70 & 29.07 & 4.63 & Acetylglutamate kinase \\
\hline NCgl2946 & 672.93 & 155.87 & 4.31 & Hypothetical protein \\
\hline NCgl1022 & 89.53 & 21.28 & 4.20 & Cysteine sulfinate desulfinase \\
\hline NCgl1023 & 368.88 & 88.67 & 4.15 & Nicotinate-nucleotide pyrophosphorylase \\
\hline NCgl1341 & 108.49 & 27.09 & 4.00 & Bifunctional ornithine acetyltransferase/N-acetylglutamate synthase \\
\hline
\end{tabular}

TABLE 2 The genes significantly down regulated by NCgl0581 deletion 


\begin{tabular}{|c|c|c|c|c|}
\hline Gene id & SSAAI $\Delta 0581$ & SSAAI & Fold change & Protein function \\
\hline NCgl0580 & 18.40 & 5152.54 & 280.02 & Hypothetical protein \\
\hline NCgl0638 & 1.71 & 20.97 & 12.22 & $\mathrm{ABC}$ transporter permease \\
\hline NCgl0639 & 11.00 & 82.47 & 7.49 & $\mathrm{ABC}$ transporter periplasmic component \\
\hline $\mathrm{NCgl} 2943$ & 207.03 & 1355.55 & 6.54 & Hypothetical protein \\
\hline NCgl0943 & 16.19 & 103.52 & 6.39 & Ara C family transcriptional regulator \\
\hline $\mathrm{NCgl0484}$ & 2.32 & 14.57 & 6.28 & $\mathrm{ABC}$ transporter permease \\
\hline $\mathrm{NCgl} 2942$ & 283.52 & 1776.15 & 6.26 & NADH: flavin oxidoreductase \\
\hline NCgl0166 & 13.41 & 79.70 & 5.94 & Hypothetical protein \\
\hline NCgl0324 & 2.11 & 11.87 & 5.61 & Zn-dependent alcohol dehydrogenase \\
\hline NCgl0282 & 5.19 & 28.25 & 5.44 & 4-Hydroxyphenyl-beta-ketoacyl-CoA hydrolase \\
\hline NCgl1975 & 102.94 & 503.75 & 4.89 & Hypothetical protein \\
\hline $\mathrm{NCgl} 2893$ & 1.25 & 6.08 & 4.84 & Efflux system protein \\
\hline NCgl0155 & 9.11 & 43.69 & 4.79 & 5-Dehydro-2-deoxygluconokinase \\
\hline NCgl0014 & 10.02 & 47.76 & 4.76 & Hypothetical protein \\
\hline $\mathrm{NCgl} 2953$ & 7.68 & 35.80 & 4.66 & Sugar permease \\
\hline $\mathrm{NCgl} 2744$ & 12.26 & 55.19 & 4.50 & Hypothetical protein \\
\hline $\mathrm{NCgl} 2970$ & 15.22 & 67.51 & 4.43 & $\mathrm{ABC}$ transporter periplasmic component \\
\hline NCgl0608 & 23.06 & 100.35 & 4.35 & $\mathrm{ABC}$ transporter permease \\
\hline NCgl0258 & 4.51 & 19.50 & 4.32 & Arsenite efflux pump ACR3 \\
\hline NCgl0281 & 16.83 & 67.69 & 4.02 & Dehydrogenase \\
\hline
\end{tabular}

The transcriptional level of serE was significantly decreased by 280 -fold following NCgl0581 deletion, revealing that $\mathrm{NCgl0581}$ is a positive regulator of serE. Furthermore, $\mathrm{NCgl0581}$ deletion downregulated the two $\mathrm{ABC}$ transporter permeases ( $\mathrm{NCgl0638}$ and $\mathrm{NCgl0484)}$ and $\mathrm{ABC}$ transporter periplasmic component (NCgl0639) by 12-, 6.3-, and 7.5-fold, respectively, and upregulated $A B C$ transporter periplasmic component (NCgl1405) by 5.88 -fold, suggesting that $\mathrm{NCgl0581}$ is involved in the synthesis of substances transported through $A B C$ transporter.

\section{Overexpression of the novel L-serine exporter system regarding to SerE and NCgl0581}

Considering that NCgI0581 could activate the expression of SerE in strain SSAAl, the overexpression of NCgl0581, serE or their co-expression was studied, and strains SSAAI-NCgI0581 and SSAAI-NCgI0581serE were constructed respectively. As shown in Fig. 4B, a decrease in cell growth was observed in SSAAI- 
NCgl0581-serE and SSAAI-NCgI0581 before $96 \mathrm{~h}$ of fermentation, and SSAAI-NCgl0581-serE showed the lowest growth rate, the time courses for L-serine production were similar in all, furthermore, the yield of Lserine to biomass (Yp/x) increased in both SSAAI-NCgl0581-serE and SSAAI-NCgI0581(Fig. 4C and 4D), which suggested that overexpression of a novel exporter SerE and it's transcriptional regulator NCgl0581 was beneficial for L-serine efflux, but not for the cell growth, with regard to Yp/x, SSAAI-NCgl0581-serE and SSAAI-NCgI0581 exhibited $9.67 \%$ and $19.17 \%$ higher value in $96 \mathrm{~h}$ respectively, when compared with SSAAI. The similar decrease in cell growth was observed in SSAAI-serE (Fig.3C), the titer of L-serine in SSAAl-serE reached $28.67 \mathrm{~g} / \mathrm{L}$, which was $10.5 \%$ higher than that noted in SSAAl. A decrease in cell growth was observed in recombinant strain, we inferred that L-serine was transported out of the cell, when L-serine was synthesized, and the intracellular L-serine was not enough for the cell growth, next we would replenish L-serine by overexpressing L-serine synthetic pathway key enzyme.

\section{Improve L-serine yield and production}

To direct more flux to L-serine synthesis, L-serine exporter SerE and L-serine synthetic pathway key enzyme (containing a feedback insensitive ser $A \triangle 197$, serB and serC encoding the deregulated 3phosphoglycerate dehydrogenase, phosphoserine phosphatase, and phosphoserine aminotransferase) were co-overexpressed in SSAAI, SSAAI-serA $\triangle 197$-serB-serC-serE had been constructed, the recombinant strain shared similar typical growth curves as the parent strain SSAAl, and reached a final L-serine titer of $32.8 \mathrm{~g} / \mathrm{L}$, which was $22.1 \%$ higher than that noted in SSAAI. Then L-serine exporter serE, serA $\triangle 197$, serB and serC were overexpressed in strain A36, and A36-serAD197-serB-serC-serE had been constructed, A36 was stemmed from SSAAl by using ARTP mutation, and it could produce $34.78 \mathrm{~g} / \mathrm{L}$ L-serine [23], As shown in Fig. 5, the tandem expression strain A36-serA $\triangle 197$-serB-serC-serE shared similar typical growth curves as the parent strain A36 in the overall process. Sucrose level decreased with time in a similar pattern for the both strains. Interestingly, when the process time in the batch cultivations exceeded $72 \mathrm{~h}$, the cell growth and L-serine titer of A36-serA $\triangle 197$-serB-serC-serE increased higher than the parent strain A36. After $120 \mathrm{~h}$ cultivation, A36-serA $\triangle 197-$ serB-serC-serE consumed all of the sucrose, and reached a final L-serine titer of $43.9 \mathrm{~g} / \mathrm{L}$, with the conversion rate of $0.44 \mathrm{~g} / \mathrm{g}$. These results demonstrate that overexpression of L-serine exporter in combination with L-serine synthetic pathway could facilitate Lserine production in C.glutamicum.

\section{Discussion}

Transport engineering is becoming an attractive strategy for strain improvement $[11,16,17]$. However, only a relatively limited number of exporters of amino acids have been identified in C. glutamicum (Table S1) $[8,12,14-17,24-27]$. In this study, SerE was identified as a novel L-serine exporter in C. glutamicum. Further analysis showed that SerE could also accept L-threonine (Fig. 2C), but not L-cysteine, similar to ThrE, which could export both L-serine and L-threonine in C. glutamicum [15]. It was assumed that the presence of $-\mathrm{OH}$ in both $\mathrm{L}$-serine and L-threonine might be the reason for these exporters to transport the two substrates. Based on homology search, SerE was found to be similar to a member of the DMT superfamily. Although DMT superfamily proteins were involved in the transport of a wide range of 
substrates, there were only a few reports available on their structures and mechanisms of substrate transport. Christian et al. performed structural and functional analyses of YddG, a DMT protein, and provided insight into the common transport mechanism shared among the DMT superfamily members [28]. It has been reported that analyses of the crystal structure data of exporters could help to elucidate the elusive transport mechanism [29], and in the future, we will plan to investigate further the SerE structures and mechanisms of substrate transport.

To explore the interaction between the known L-serine exporter ThrE and the novel exporter SerE on Lserine accept, ThrE and SerE single and double mutants were constructed. The results showed that serE and thrE double deletion mutant could still accumulate $10.34 \mathrm{~g} / \mathrm{L} \mathrm{L-serine} \mathrm{(Fig.} \mathrm{3B),} \mathrm{suggesting} \mathrm{that} \mathrm{C.}$ glutamicum might also possess other L-serine exporter systems. The evolution of multiple exporter systems for a single substrate is beneficial for the survival of bacteria in variable environment $[7,30]$. It must be noted that overexpression of serE in SSAAI resulted in $10.5 \%$ increase in L-serine titer, but a decrease in cell growth. This could be due to the use of constitutive-type promoter to overexpress SerE, which resulted in higher L-serine efflux. As sufficient L-serine content is important to maintain cell growth, a decrease in cell growth was noted as a stress response to serE overexpression. In future studies, better tuning of the serE expression will be performed in SSAAI by testing different promoters and RBS. When thrE and serE were co-overexpressed in SSAAI, SSAAl-thrE-serE exhibited a lower cell growth than SSAAl, and similar L-serine titer with SSAAI-serE was observed (Fig. 3C). A severe decline in cell growth was observed in all exporter overexpression strains, it was likely that this may be caused by accumulation of L-serine in the media as well as the additional burden on the cell of overexpressing the exporters, the similar result was observed by Mundhada et,al[31].

NCgl0581 was identified as a novel L-serine exporter SerE's transcriptional regulator, and EMSA was performed to confirm the binding sites of NCgl0581 with the promoter of SerE. A previous study reported that the first member of the protein-gene pairs, ArgP-argO in E. coli and LysG-lysE in C. glutamicum, is a LysR-type transcriptional regulator, while the second member is its target gene encoding an amino acid exporter [22, 32, 33]. Similarly, NCgl0581-serE might also be a protein-gene pair sharing the same regulation mechanism. A serine-biosensor based NCgl0581 was reported by Binder et al.[34], and based on this study, we constructed a biosensor for L-serine and found that NCgl0581 activated NCgl0580 (SerE) expression in the presence of L-serine, the expression of SerE enhancing with the L-serine titer increasing [35]. However, NCgl0581 did not activate the expression of SerE in the presence of L-alanine and $L$-valine. To further confirm whether SerE could accept L-alanine and L-valine, peptide feeding assays were employed using dipeptides (Ala-Ala, Val-Val) with SSAAI and SSAAI $\triangle$ serE. The results revealed that SerE could neither export L-alanine nor L-valine (data not shown). Moreover, transcriptome sequencing showed that NCgl0581 regulated 115 genes in C. glutamicum, suggesting that NCgl0581 was a novel global transcriptional regulator in C. glutamicum. Transcriptional regulators and their roles in expression control of target genes are important for metabolic engineering of $\mathrm{C}$. glutamicum for industrial applications [36], and this study provided a new member of transcriptional regulator family. 
When L-serine exporter SerE was overexpressed alone, which resulted in increase of $10.8 \%$ of L-serine titer, at same time, a decrease in cell growth was observed in recombinant strain, we inferred that L-serine was transported out of the cell, when L-serine was synthesized, and the intracellular L-serine was not enough for the cell growth, to replenish L-serine by overexpressing L-serine synthetic pathway key enzyme, the cell growth was restored, and L-serine titer was increased to $43.9 \mathrm{~g} / \mathrm{L}$, and L-serine yield was $0.44 \mathrm{~g} / \mathrm{g}$ sucrose, which were the highest titer and yield reported so far for any organism. The results indicated that serA $\triangle 197$, serC, serB overexpression ensured sufficient L-serine supply avoided the cell growth inhibition. In Mundhada's study, $37 \mathrm{~g} / \mathrm{L}$ of L-serine was produced with a yield of $0.24 \mathrm{~g} / \mathrm{g}$ glucose in E. coli [2], and in their previous study, L-serine titer was $11.7 \mathrm{~g} / \mathrm{L}$ with highest yield of $0.43 \mathrm{~g} / \mathrm{g}$ glucose [31]. Interestingly, we found that L-serine titer increased significantly by overexpressing serB in A36, with L-serine titer of $37.9 \mathrm{~g} / \mathrm{L}$, which was $24 \%$ higher than that of $A 36$, serB encoded phosphoserine phosphatase (PSP, EC 3.1.3.3), catalyzed the last step of L-serine biosynthesis, however, L-serine titer had not significantly change when serA $\triangle 197$, serC was overexpressed in A36 respectively (with L-serine titer of $31.1 \mathrm{~g} / \mathrm{L}$ and $32.78 \mathrm{~g} / \mathrm{L}$ ) (Fig.S5), and these results were in consistent with the previous report completely, in which overexpression of the mutant allele serA $\Delta 197$ in C. glutamicum13032 either alone or in combination with overexpression of serC and serB did not result in significant L-serine accumulation [3],which might be due to the poor accumulation and intracellular conversion of L-serine in $\mathrm{C}$.

glutamicum13032. In a recent study, $50 \mathrm{~g} / \mathrm{L}$ of L-serine was produced with glucose as carbon source in $\mathrm{E}$. coli, with a yield of $0.26-0.30 \mathrm{~g} / \mathrm{g}$ glucose [37], in which $50 \mathrm{~g} / \mathrm{L}$ is the highest reported so far for L-serine production, however, the yield was also lower than our present study $(0.44 \mathrm{~g} / \mathrm{g}$ sucrose). It is possible that fine controlling the three enzymes of the L-serine biosynthesis pathway to enhance L-serine production further.

\section{Conclusion}

In the present work, a novel exporter SerE and its positive regulator NCgl0581 were identified in C. glutamicum, with SerE also exhibiting the ability to accept L-threonine and NCgl0581 acting as a novel global transcriptional regulator in C. glutamicum, and by overexpressing novel exporter combined with Lserine synthetic pathway enzyme, increased L-serine yield significantly. These results enrich our understanding of amino acid transport and can provide additional targets for exporter engineering in $\mathrm{C}$. glutamicum.

\section{Materials And Methods}

\section{Strains, plasmids, and growth conditions}

The strains and plasmids used in this study are listed in Table 3. E. coli JM109 was used as the cloning host, and was grown in lysogeny broth (LB) medium (containing $5.0 \mathrm{~g} / \mathrm{L}$ yeast extract, $10.0 \mathrm{~g} / \mathrm{L}$ tryptone, and $10.0 \mathrm{~g} / \mathrm{L} \mathrm{NaCl}$ ) at $37^{\circ} \mathrm{C}$ and $220 \mathrm{rpm}$. The engineered SSAAI (CGMCC No.15170) was constructed in our laboratory by knocking out 591 bp of the C-terminal domain of $\operatorname{ser} A$, deleting $s d a A$, avtA, and alaT, as well as attenuating ilvBN in the genome of $C$. glutamicum SYPS-062-33a (CGMCC No. 8667). The seed 
and fermentation media for $C$. glutamicum were prepared as described previously [5]. The $C$. glutamicum strains were pre-incubated in the seed medium overnight to an optical density $\left(\mathrm{OD}_{562}\right)$ of about 25 , and then inoculated at an initial concentration of $\mathrm{OD}_{562}=1$ into a $250 \mathrm{~mL}$ flask containing $25 \mathrm{~mL}$ of the fermentation medium at $30^{\circ} \mathrm{C}$ and $120 \mathrm{rpm}$. The antibiotic kanamycin $(50 \mathrm{mg} / \mathrm{L})$ was added when necessary. Samples were withdrawn periodically for the measurement of residual sugar, amino acids, and $\mathrm{OD}_{562}$ as described previously [5].

TABLE 3 Strains and plasmids used in this study. 
E. coli

JM109

C. glutamicum

SSAAI

A36

SSAAI-thrE

SSAAI $\Delta$ thrE

SSAAI $\Delta \mathrm{NCgl} 2050$

SSAAI $\Delta \mathrm{NCgl} 2065$

SSAAI $\Delta$ NCgl0580

SSAAI-10

SSAAI-egfp

SSAAI-serE-egfp

SSAAI-NCgl0581

SSAAI-NCgl0581-serE

SSAAI $\Delta$ NCgl0581

SSAAI $\Delta$ NCgl0581-1

SSAAI $\Delta$ NCgl0581-0

SSAAI $\Delta$ NCgl0580-serE

SSAAI-serE

ATCC13032

ATCC13032 $\Delta$ serE

pK18mobsacB

pK18mobsacBsthrE
recA1, endA1, gyrA96, thi-1, hsdR17, supE44, relA1

Laboratory

strain

C. glutamicum SYPS-33a with deletion of the 591 bp in the C-terminus of serA, deletion of sdaA,alaT,avta and attenuation ofilvBN

SSAAI mutant strain

SSAAI harboring plasmid pDXW-10-thrE

This study

SSAAI with deletion of thrE

This study

SSAAI with deletion of NCgl2050

This study

SSAAI with deletion of NCgl2065

This study

SSAAI with deletion of NCgl0580

This study

SSAAI harboring plasmid pDXW-10

This study

SSAAI harboring plasmid pDXW-10-egfp

This study

SSAAI harboring plasmid pDXW-10-serE-egfp

This study

SSAAI harboring plasmid pDXW-10- NCgl0581

This study

SSAAI harboring plasmid pDXW-10- NCgl0581-serE

This study

SSAAI with deletion of NCgl0581

This study

SSAAI $\Delta$ NCgl0581 harboring pDXW-11-1

This study

SSAAI $\Delta$ NCgl0581 harboring pDXW-11-0

This study

SSAAI $\Delta$ serE harboring plasmid pDXW-10-serE (NCgl0580)

This study

SSAAI harboring plasmid pDXW-10-serE (NCgl0580)

This study

Wild type

Laboratory

strain

ATCC13032 with deletion of serE (NCgl0580)

This study

Integration vector, ori $V$, oriT, mob, $s a c B, \mathrm{Km}^{\mathrm{r}}$

This study 
pK18mobsacB $\Delta$ NCgl2050pK18mobsacB carrying the up- and downstream homologous fragments of NCgl2050 gene This study for NCgl2050 deletion

pK18mobsacBANCgl2065pK18mobsacB carrying the up- and downstream homologous fragments of NCgl2065 gene This study for NCgl2065 deletion

pK18mobsacB $\operatorname{NCgl0580pK18mobsacB~carrying~the~up-~and~downstream~homologous~fragments~of~NCgl0580~gene~This~study~}$ for NCgl0580 deletion

pK18mobsacB $\Delta$ NCgl0581pK18mobsacB carrying the up- and downstream homologous fragments of NCgl0581 gene This study for NCgl0581 deletion

pDXW-10

pDXW-10-thrE

pDXW-10-serE

pDXW-10-egfp

pDXW-10-egfp-serE

pDXW-10- NCgl0581

pDXW-10- NCgl0581-

serE

pDXW-11

pDXW-11-1

pDXW-11-0
E. coli- C. glutamicum shuttle vector, tacM promoter, $\mathrm{Km}^{\mathrm{r}}$

pDXW-10 carrying the gene of thrE

pDXW-10 carrying the gene of serE

pDXW-10 carrying the gene of egfp

pDXW-10 carrying the gene of egfp and serE for the expression of fusion protein EGFP-

SerE

pDXW-10 carrying the gene of NCgl0581

This study

pDXW-10 carrying the gene of NCgl0581 and serE

This study

pDXW-11 carrying the fragments of NCgl0581, the intergenic region between NCgl0581

This study and NCgl0580, and egfp

pDXW-11 carrying the fragments of the intergenic region between NCgl0581 and

This study

$\mathrm{Km}^{\mathrm{r}}$, kanamycin resistance.

\section{Construction of plasmids and strains}

The primers used in this study for gene expression/deletion are listed in Table S2. Gene deletion was performed using the nonreplicable deletion vector pK18mobsacB, as reported previously [38]. For example, to achieve thrE deletion, the homologous-arm fragments for thrE deletion were amplified from SSAAI chromosome using the primer pairs thrE1/2 for the upstream fragment and thrE3/4 for the downstream fragment. Then, with the two fragments as templates, a crossover PCR was performed using 
the primer pair thrE1/4. The truncated product of thrE was digested with $X b a \rrbracket$ and $H i n d \rrbracket$ and ligated to the vector pK $18 \mathrm{mob} s a c B$ that was similarly treated. The recombinant plasmid pK18mobsacB $\triangle$ thrE was transformed into SSAAI competent cells by electroporation, and chromosomal deletion was performed by selecting cells that were kanamycin resistant and sucrose nonresistant, and verified by PCR.

The pDXW-10 and pDXW-11 plasmids were used to overexpress genes in C. glutamicum [39, 40]. The recombinant plasmids were constructed as follows: the genes thrE and serE were amplified, digested, and

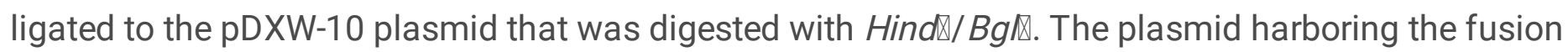
protein, SerE-EGFP (enhanced green fluorescent protein) was constructed by using the method reported in a previous study [19]. To confirm the role of NCgI0581 on NCgl0580 expression, the fragment consisting of intergenic region of NCgl0581 and NCgl0580 and EGFP with or without NCgI0581 was ligated to the plasmid pDXW-11 by Clon Express MultiS One Step Cloning Kit (Vazyme, Nanjing, China). The strains were constructed by electroporation with the corresponding plasmids.

The genes, serA $\triangle 197$, serB, and serC, were PCR amplified from SSAAI using primers shown in Table S2. To construct plasmid pDXW-10-serA $\triangle 197$, the resultant fragment of serA $\triangle 197$ was digested with EcoRI and Notl and cloned into pDXW-10. To construct plasmid pDXW-10-serA 1 197-serB-serC, PCR fragments of $\operatorname{ser} B$, and $\operatorname{ser} C$ were digested with the appropriate restriction enzymes and successively cloned into the corresponding plasmids to form plasmid pDXW-10-serA $\triangle 197$-serB-serC. The resulting plasmid (pDXW-10serA $\triangle 197$-serB-serC) was then subjected to double digestion by $N d e l$ and Pacl for cloning of NCgl0580 to form pDXW-10-serA $\triangle 197$-serB-serC-NCgI0580.

\section{Confocal microscopic observation}

The strains SSAAI-10 (SSAAI harboring plasmid pDXW-10), SSAAl-egfp, and SSAAI-serE-egfp were grown in the seed medium and harvested during the exponential phase. The cells were washed twice and maintained in PBS ( $\mathrm{pH} 7.4)$, mounted on a microscope slide, and observed under a Leica laser scanning confocal microscope (Leica, TCS SP8; Leica, Wetzlar, Germany) equipped with a HC PL Apo 63x/1.40 Oil CS2 oil-Immersion objective, with excitation filter at $488 \mathrm{~nm}$ and emission filter at 510-550 nm. The digital images were acquired and analyzed with Lecia Application Suite X 2.0.

\section{Membrane and cytoplasmic protein extraction and fluorescence measurements}

The strains SSAAI-10, SSAAI-egfp, and SSAAI-serE-egfp were used for extracting membrane and cytoplasmic proteins to determine SerE localization. The extraction was performed using Membrane and a Cytoplasmic Protein Extraction Kit according to the manufacturer's protocol (Beyotime, Nanjing, China). The cells were washed twice with PBS ( $\mathrm{pH}$ 7.4) and disrupted by ultrasonication on ice (pulse, $4 \mathrm{~s}$; interval, $6 \mathrm{~s}$; total duration, $30 \mathrm{~min}$ ) (Sonics Vibra-Cell ${ }^{\mathrm{m}}$, Sonics, Newtown, CT, USA). The supernatant containing cytoplasmic proteins was collected by centrifugation $\left(700 \times g, 4^{\circ} \mathrm{C}\right.$ for $\left.10 \mathrm{~min}\right)$, and the precipitate was used for extracting membrane proteins. The protein concentration was determined with a Modified BCA Protein Assay Kit (Sangon, China). After extraction, the fluorescence intensity (excitation at 
$488 \mathrm{~nm}$, emission at $517 \mathrm{~nm}$ ) of the membrane and cytoplasmic proteins was determined using fluorescence spectrophotometer (Synergy H4; BioTek, Winooski, VT, USA).

\section{Amino acid export assay}

For ascertaining the function of serE, a dipeptide Ser-Ser addition assay was conducted [15]. In brief, preincubated cells (in seed medium) were washed once with CGX『 minimal medium [41], inoculated into $\mathrm{CGX} \rrbracket$ minimal medium with $2 \mathrm{mM}$ Ser-Ser (other dipeptide), and incubated for $2 \mathrm{~h}$ at $30^{\circ} \mathrm{C}$. Then, the cells were harvested, washed once with cold CGX『 minimal medium, and resuspended in CGX》 minimal medium. Amino acid excretion was initiated by adding $2 \mathrm{mM}$ Ser-Ser (another dipeptide). HPLC was used to determine the concentrations of amino acids [19].

\section{Analytical procedures}

Cell density $\left(\mathrm{OD}_{562}\right)$ was measured using an AOE UV-1200S UV/vis spectrophotometer (AOE Instruments Co. Inc., Shanghai, China). Glucose concentration was determined using SBA-40E glucose analyzer (Biology Institute of Shandong Academy of Sciences, China). For measurement of extracellular L-serine concentration in shake-flask fermentation, $500 \mu \mathrm{L}$ of the culture were centrifuged at $700 \times g$ for 5 min, and the supernatant was used for detection after appropriate dilution. To ascertain intracellular L-serine concentration, $300 \mu \mathrm{L}$ of the culture were centrifuged at $700 \times g$ and $4^{\circ} \mathrm{C}$ for $10 \mathrm{~min}$, and $300 \mu \mathrm{L}$ of water were added to the cells. The cells were disrupted by FastPrep-24 5G instrument $(5 \mathrm{~m} / \mathrm{s}, 120 \mathrm{~s}$, MP Biomedicals, Shanghai, China). The cytoplasmic volume was assumed to be $2 \mu \mathrm{L} / \mathrm{mg}$ dry cell weight [27]. The titers of intracellular and extracellular L-serine and other amino acids were analyzed by HPLC using phenyl isothiocyanate as a precolumn derivatization agent, according to our previously study [8].

\section{EMSA}

To identify the binding site of NCgI0581 in the NCgI0580 promoter region, EMSA was conducted by using Non-Radioactive EMSA Kits with Biotin-Probes User's Manual VER. 5.11 (Viagene Biotech Inc, Changzhou, China), according to the manufacturer's instruction. The consensus oligonucleotides were BIO-JNZXM-TP (5'-AAACAGCCAA CTATAGTTAAGTAATA-3') and BIO-JNZXM-BM (5'-TATTACTTAACTATAGTTGGCTGTTT$\left.3^{\prime}\right)$.

\section{Supplementary Information}

Supplementary information accompanies this paper.

\section{Abbreviations}

C.glutamicum: Corynebacterium glutamicum®SSAAI : C. glutamicum $\triangle$ SSAAI $₫ E G F P \bowtie E n h a n c e d ~ g r e e n$ fluorescent protein $\triangle \mathrm{EMSA} \triangle \mathrm{Electrophoretic} \mathrm{mobility} \mathrm{shift} \mathrm{assays} \triangle \mathrm{DMT} \otimes T$ The drug/metabolite transporter

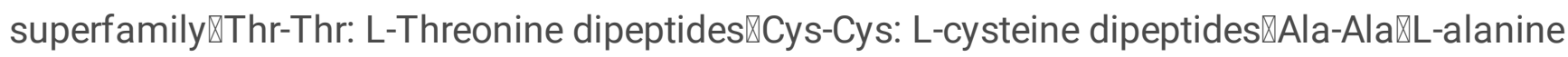




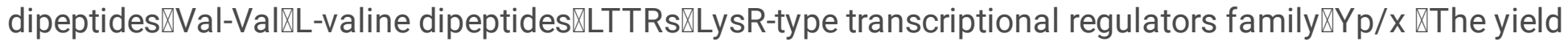

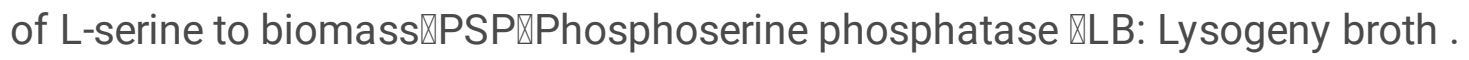

\section{Declarations}

\section{Authors' contributions}

$X M Z, Y J G$ and ZW C conceived and designed the experiments. XM Z led the performance of the experiments, YJ G and ZW C performed the experiments. XM Z, GQ X and XJ Z analyzed the data. XM Z, YJ G and ZW C wrote the manuscript, JS S, MK and ZH X gave some suggestions for the experiments and revised the manuscript. All authors read and approved the final manuscript

\section{Competing interests}

The authors declare that they have no competing interests.

\section{Availability of data and materials}

The datasets used and analyzed during this study are available from the corresponding author on reasonable request.

\section{Consent for publication}

Not applicable.

\section{Ethics approval and consent to participate}

Not applicable.

\section{Funding}

This work was financially supported by the National Key Research and Development Program of China (2018YFA0901400). The National Natural Science Foundation of China (No. 31600044). Program of National First-class Discipline Program of Light Industry Technology and Engineering (LITE2018-11). Program of Introducing Talents of Discipline to Universities (No.111-2-06). International Joint Research Laboratory for Engineering Synthetic Biosystem for Intelligent Biomanufacturing at Jiangnan University. Top-notch Academic Programs Project of Jiangsu Higher Education Institutions (TAPP). And China Postdoctoral Science Foundation (No.2018M640452).

\section{References}


1. Zhang XM, Xu GQ, Shi JS, Koffas MAG, Xu ZH: Microbial production of L-serine from renewable feedstocks. Trends in Biotechnology 2018, 36:700-712.

2. Mundhada H, Seoane JM, Schneider K, Koza A, Christensen HB, Klein T, Phaneuf PV, Herrgard M, Feist AM, Nielsen AT: Increased production of L-serine in Escherichia coil through adaptive laboratory evolution. Metabolic Engineering 2017, 39:141-150.

3. Peters-Wendisch P, Stolz M, Etterich H, Kennerknecht N, Sahm H, Eggeling L: Metabolic engineering of Corynebacterium glutamicum for L-serine production. Applied and Environmental Microbiology 2005, 71:7139-7144.

4. Stolz M, Peters-Wendisch P, Etterich H, Gerharz T, Faurie R, Sahm H, Fersterra H, Eggeling L: Reduced folate supply as a key to enhanced L-serine production by Corynebacterium glutamicum. Applied and Environmental Microbiology 2007, 73:750-755.

5. Zhu Q, Zhang X, Luo Y, Guo W, Xu G, Shi J, Xu Z: L-Serine overproduction with minimization of byproduct synthesis by engineered Corynebacterium glutamicum. Applied Microbiology \& Biotechnology 2015, 99:1665-1673.

6. Xu G, Jin X, Wen G, Dou W, Zhang X, Xu Z: Characterization, modification, and overexpression of 3phosphoglycerate dehydrogenase in Corynebacterium glutamicum for enhancing L-serine production. Annals of Microbiology 2015, 65:929-935.

7. Jones CM, Lozada NJH, Pfleger BF: Efflux systems in bacteria and their metabolic engineering applications. Applied Microbiology and Biotechnology 2015, 99:9381-9393.

8. Eggeling L, Sahm H: New ubiquitous translocators: amino acid export by Corynebacterium glutamicum and Escherichia coli. Archives of Microbiology 2003, 180:155-160.

9. Dunlop MJ, Dossani ZY, Szmidt HL, Chu HC, Lee TS, Keasling JD, Hadi MZ, Mukhopadhyay A: Engineering microbial biofuel tolerance and export using efflux pumps. Molecular Systems Biology 2011, 7:7.

10. Kim S, Ihara K, Katsube S, Hori H, Ando T, Isogai E, Yoneyama H: Characterization of the L-alanine exporter AlaE of Escherichia coli and its potential role in protecting cells from a toxic-level accumulation of L-alanine and its derivatives. Microbiologyopen 2015, 4:632-643.

11. Pérez-García F, Wendisch VF: Transport and metabolic engineering of the cell factory Corynebacterium glutamicum. Fems Microbiology Letters 2018.

12. Vrljic M, Sahm H, Eggeling L: A new type of transporter with a new type of cellular function: L-lysine export from Corynebacterium glutamicum. Molecular Microbiology 2010, 22:815-826.

13. Dassler T, Maier T, Winterhalter $C$, Bock A: Identification of a major facilitator protein from Escherichia coli involved in efflux of metabolites of the cysteine pathway. Molecular Microbiology 2000, 36:1101-1112.

14. Jun N, Seiko H, Hisao I, Masaaki W: Mutations of the Corynebacterium glutamicum NCgl1221 gene, encoding a mechanosensitive channel homolog, induce L-glutamic acid production. Appl Environ Microbiol 2007, 73:4491-4498. 
15. Simic P, Sahm H, Eggeling L: L-threonine export: use of peptides to identify a new translocator from Corynebacterium glutamicum. Journal of Bacteriology 2001, 183:5317-5324.

16. Lubitz D, Jorge JMP, Pérez-García F, Taniguchi H, Wendisch VF: Roles of export genes cgmA and lysE for the production of L-arginine and L-citrulline by Corynebacterium glutamicum. Applied Microbiology \& Biotechnology 2016, 100:1-10.

17. Schwede T: Protein Modeling: What happened to the "protein structure gap"? Structure 2013, 21:1531-1540.

18. Petra S, Juliane W, Hermann S, Lothar E: Identification of glyA (encoding serine hydroxymethyltransferase) and its use together with the exporter ThrE to increase L-threonine accumulation by Corynebacterium glutamicum. Applied \& Environmental Microbiology 2002, 68:3321.

19. Liu Q, Liang Y, Zhang Y, Shang X, Liu S, Wen J, Wen T: YjeH is a novel exporter of L-methionine and branched-chain amino acids in Escherichia coli. Applied \& Environmental Microbiology 2015, 81:7753.

20. Huhn S, Jolkver E, Kramer R, Marin K: Identification of the membrane protein SucE and its role in succinate transport in Corynebacterium glutamicum. Applied Microbiology and Biotechnology 2011, 89:327-335.

21. Maddocks SE, Oyston PCF: Structure and function of the LysR-type transcriptional regulator (LTTR) family proteins. Microbiology 2008, 154:3609-3623.

22. Bellmann A, Vrljic M, Patek M, Sahm H, Kramer R, Eggeling L: Expression control and specificity of the basic amino acid exporter LysE of Corynebacterium glutamicum. Microbiology-Sgm 2001, 147:1765-1774.

23. Xin Z, Xiaomei Z, Guoqiang X, Xiaojuan Z, Jinsong S, Zhenghong X: Integration of ARTP mutagenesis with biosensor-mediated high-throughput screening to improve L-serine yield in Corynebacterium glutamicum. Applied Microbiology and Biotechnology 2018, 102:5939-5951.

24. Xiuling S, Yun Z, Guoqiang Z, Xin C, Aihua D, Yong L, Tingyi W: Characterization and Molecular Mechanism of AroP as an Aromatic Amino Acid and Histidine Transporter in Corynebacterium glutamicum. Journal of Bacteriology 2013, 195:5334-5342.

25. Nicole K, Hermann S, Ming-Ren Y, Miroslav P, Jr MH, Saier, Lothar E: Export of L-isoleucine from Corynebacterium glutamicum: a two-gene-encoded member of a new translocator family. Journal of Bacteriology 2002, 184:3947-3956.

26. Wang Y, Cao G, Xu D, Fan L, Wu X, Ni X, Zhao S, Zheng P, Sun J, Ma Y: A novel L-glutamate exporter of Corynebacterium glutamicum. Applied \& Environmental Microbiology 2018, 84: 02691.

27. Zhi Z, Jiu-Yuan D, Tang L, Ning-Yi Z, Shuang-Jiang L: The ncgl1108 (PheP (Cg)) gene encodes a new L-phe transporter in Corynebacterium glutamicum. Applied Microbiology \& Biotechnology 2011, 90:2005-2013.

28. Christian TT, Dietrich D, Brigitte B, Andreas B, Reinhard KM: Characterization of methionine export in Corynebacterium glutamicum. Journal of Bacteriology 2005, 187:3786. 
29. Jie Y, Jingpeng G, Johanna H, Erwin S, Maojun Y: Structural basis for substrate specificity of an amino acid ABC transporter. Proceedings of the National Academy of Sciences of the United States of America 2015, 112:5243-5248.

30. Eggeling L: Exporters for production of amino acids and other small molecules. Adv Biochem Eng Biotechnol 2016, 159:199-225.

31. Mundhada H, Schneider K, Christensen HB, Nielsen AT: Engineering of high yield production of Lserine in Escherichia coli. Biotechnology and Bioengineering 2016, 113:807-816.

32. Nandineni MR, Gowrishankar J: Evidence for an arginine exporter encoded by $y g g A(\arg 0)$ that is regulated by the LysR-type transcriptional regulator ArgP in Escherichia coli. Journal of Bacteriology 2004, 186:3539-3546.

33. Marbaniang $\mathrm{CN}$, Gowrishankar J: Transcriptional cross-regulation between gram-negative and grampositive bacteria, demonstrated using ArgP-argO of Escherichia coli and LysG-lysE of Corynebacterium glutamicum. Journal of Bacteriology 2012, 194:5657-5666.

34. Binder S: A high-throughput approach to identify genomic variants of bacterial metabolite producers at the single-cell level. Genome Biology,13,5(2012-05-28) 2012, 13:R40.

35. Zhang X, Zhang X, Xu G, Zhang X, Shi J, Xu Z: Integration of ARTP mutagenesis with biosensormediated high-throughput screening to improve L-serine yield in Corynebacterium glutamicum. Applied Microbiology \& Biotechnology 2018, 102:1-13.

36. Shah A, Blombach B, Gauttam R, Eikmanns BJ: The RamA regulon: complex regulatory interactions in relation to central metabolism in Corynebacterium glutamicum. Applied Microbiology \& Biotechnology 2018, 102:1-10.

37. Rennig M, Mundhada H, Wordofa GG, Gerngross D, Wulff T, Worberg A, Nielsen AT, Norholm MHH: Industrializing a bacterial strain for L-serine production through translation initiation optimization. ACS synthetic biology 2019,8:2347-2358.

38. Schafer A, Tauch A, Jager W, Kalinowski J, Thierbach G, Puhler A: Small mobilizable multipurpose cloning vectors derived from the escherichia-coli plasmids pk18 and pk19-selection of defined deletions in the chromosome of Corynebacterium glutamicum. Gene 1994, 145:69-73.

39. Xu D, Tan Y, Li Y, Wang X: Construction of a novel promoter-probe vector and its application for screening strong promoter for brevibacterium flavum metabolic engineering. World Journal of Microbiology \& Biotechnology 2011, 27:961-968.

40. Xu DQ, Tan YZ, Shi F, Wang XY: An improved shuttle vector constructed for metabolic engineering research in Corynebacterium glutamicum. Plasmid 2010, 64:85-91.

41. Keilhauer C, Eggeling L, Sahm H: Isoleucine synthesis in Corynebacterium glutamicum: molecular analysis of the ilvB-ilvN-ilvC operon. Journal of Bacteriology 1993, 175:5595.

\section{Figures}



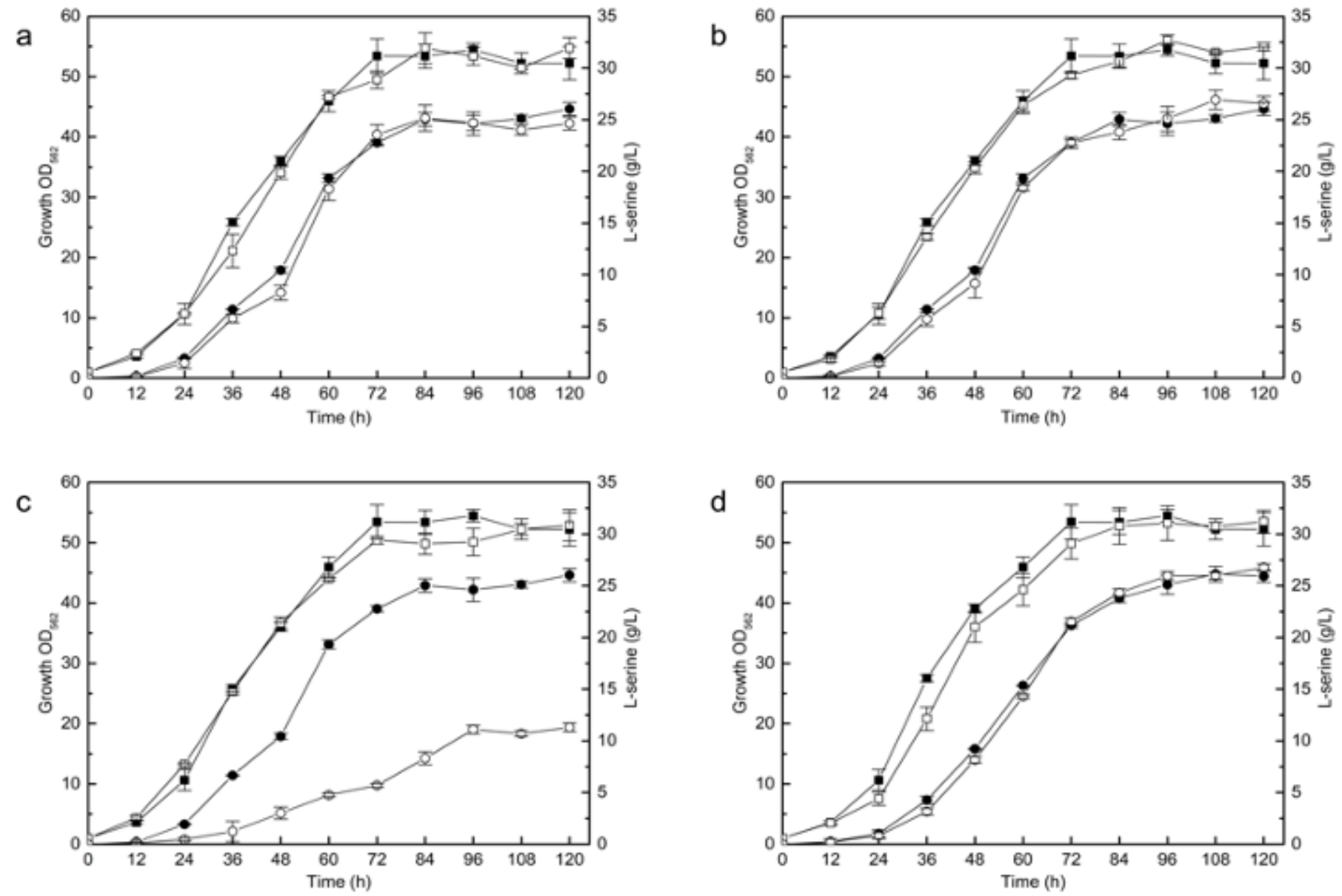

Figure 1

Effect of NCgl2050, NCgl2065, NCgl0580 deletion and plasmid compensating strain on SSAAl. (a) NCgl2050 deletion strain SSAAI NCgl2050 (open symbols), SSAAI (solid symbols). (b) NCgl2065 deletion strain SSAAI $\triangle \mathrm{NCgl} 2065$ (open symbols), SSAAl (solid symbols). (c) NCgl0580 deletion strain SSAAI $\triangle N C g I 0580$ (open symbols), SSAAI (solid symbols). (d) The plasmid compensating strain SSAAI $\triangle$ N0580-NCgI0580 (open symbols). SSAAI (solid symbols). Squares and circles indicate the cell growth OD562 and L-serine titer, respectively. 


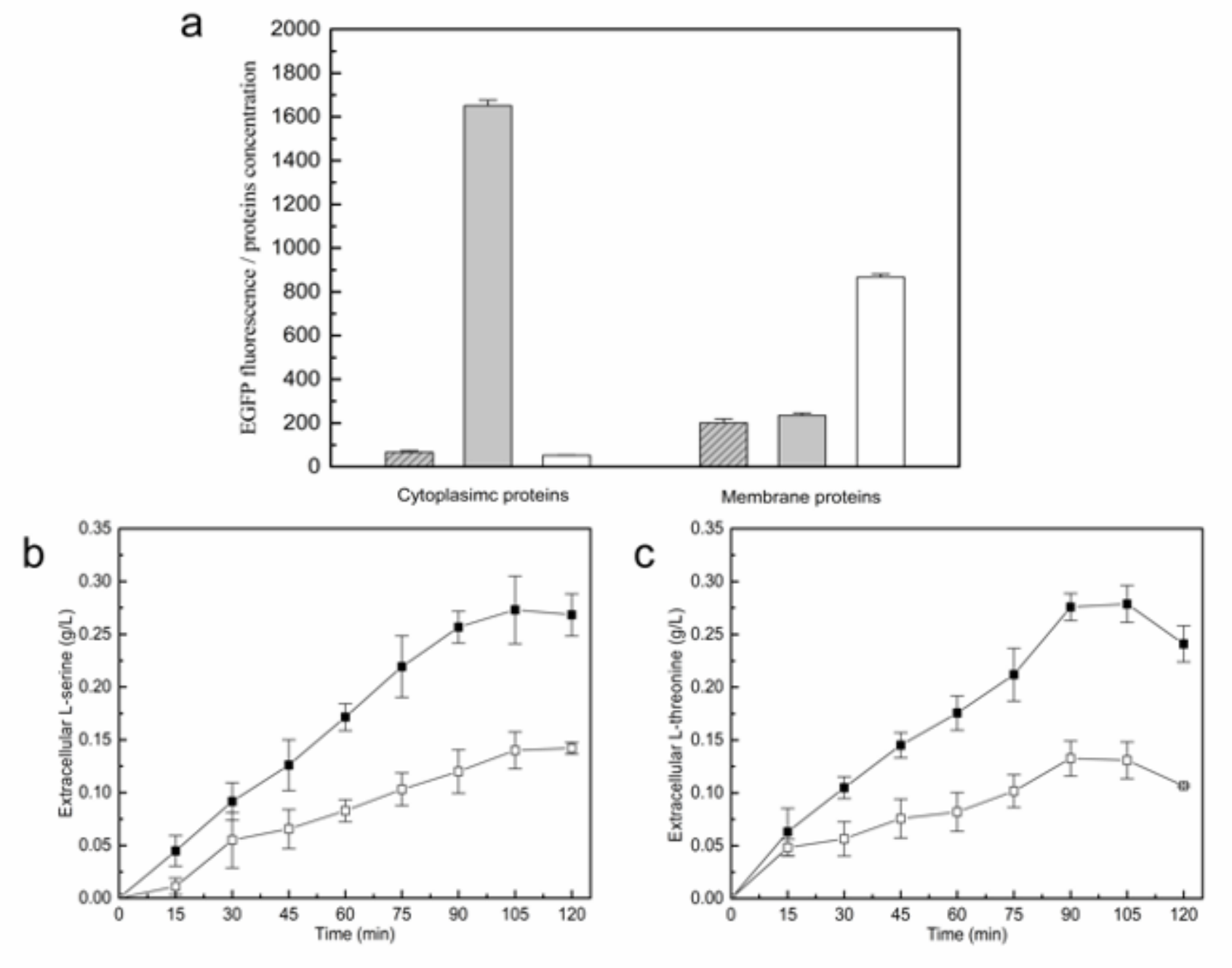

Figure 2

The fluorescence of cytoplasmic proteins and membrane proteins and the result of amino acid export of SerE by using peptide feeding approach in SSAAI. (a) The fluorescence of cytoplasmic proteins and membrane proteins of SSAAI-10 (SSAAI harboring plasmid pDXW-10 only, grey bar with slash), SSAAIegfp (SSAAI overexpressing EGFP protein with pDXW-10, grey bar) and SSAAI-serE-egfp (SSAAI overexpressing SerE-EGFP fusion protein with pDXW-10, white bar). (b) Extracellular concentration of Lserine in SSAAI (solid symbols) and serE deletion strain SSAAI $\triangle$ serE (open symbols). (c) Extracellular concentration of L-threonine in SSAAI (squares) and serE deletion strain SSAAI $\triangle$ serE (circles). 

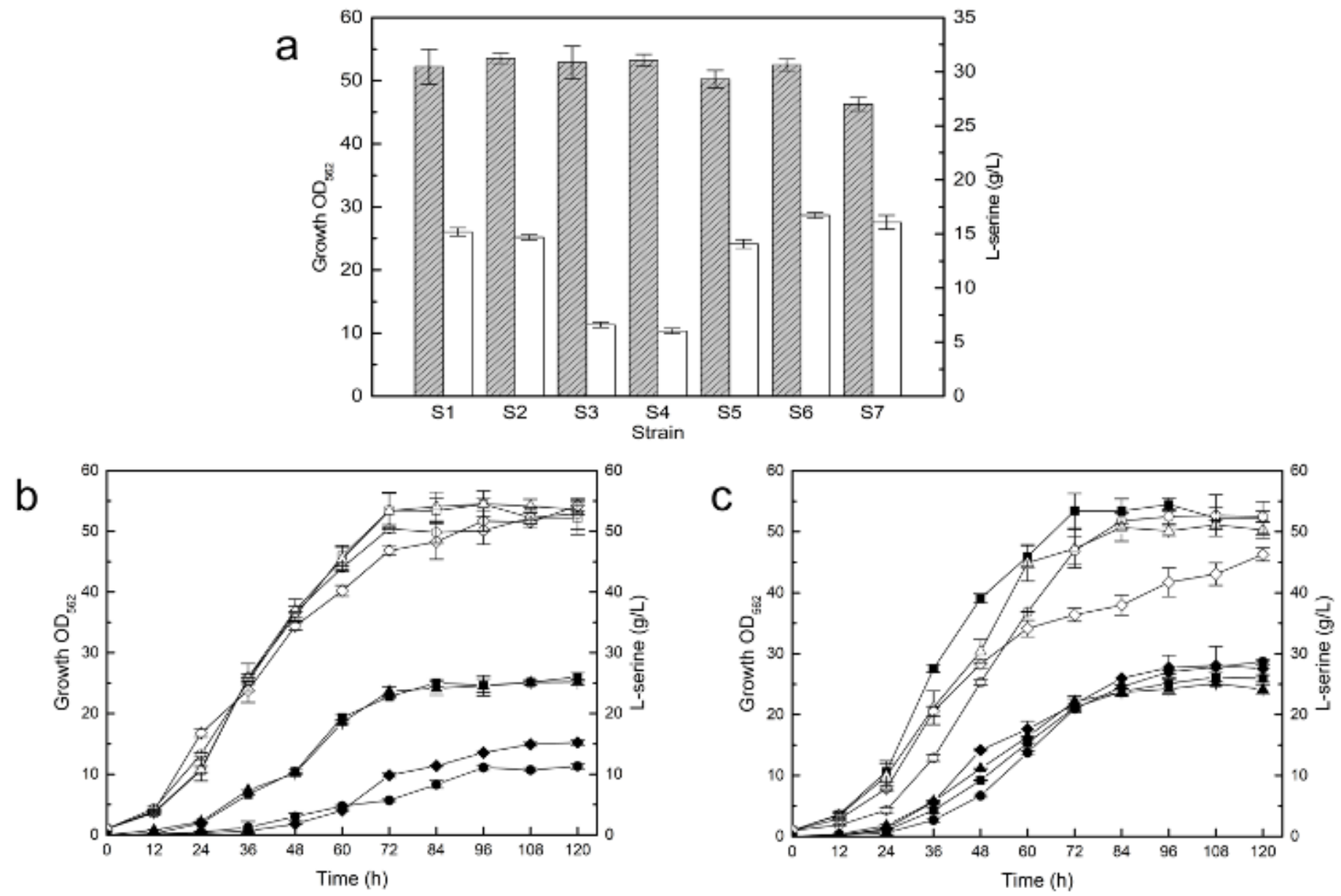

Figure 3

The effect of the exporter thrE, serE deletion or overexpression on strain SSAAI. (a)The cell growth (grey bar with slash), L-serine titer (white bar). SSAAI (S1), thrE deletion strain SSAAI $\triangle$ thrE (S2), serE deletion strain SSAAI $\Delta$ serE (S3), thrE and serE deletion strain SSAAI $\Delta$ serE $\Delta$ thrE (S4), thrE overexpression strain SSAAl-thrE (S5), serE overexpression strain SSAAl-serE (S6), thrE and serE double overexpression strain SSAAI-serE -thrE (S7). (b) The cell growth (open symbols) and L-serine titer (solid symbols) of stain SSAAI (squares), serE deletion strain SSAAI $\Delta$ serE (circles), thrE deletion strain SSAAI $\Delta$ thrE (triangles), thrE and serE deletion strain SSAAI $\Delta$ serE $\Delta$ thrE (rhombus). (c) The cell growth (open symbols) and L-serine titer (solid symbols) of stain SSAAI (squares), serE overexpression strain SSAAI-serE (circles), thrE overexpression strain SSAAI-thrE (triangles) and thrE and serE double overexpression strain SSAAI-serE thrE (rhombus). 

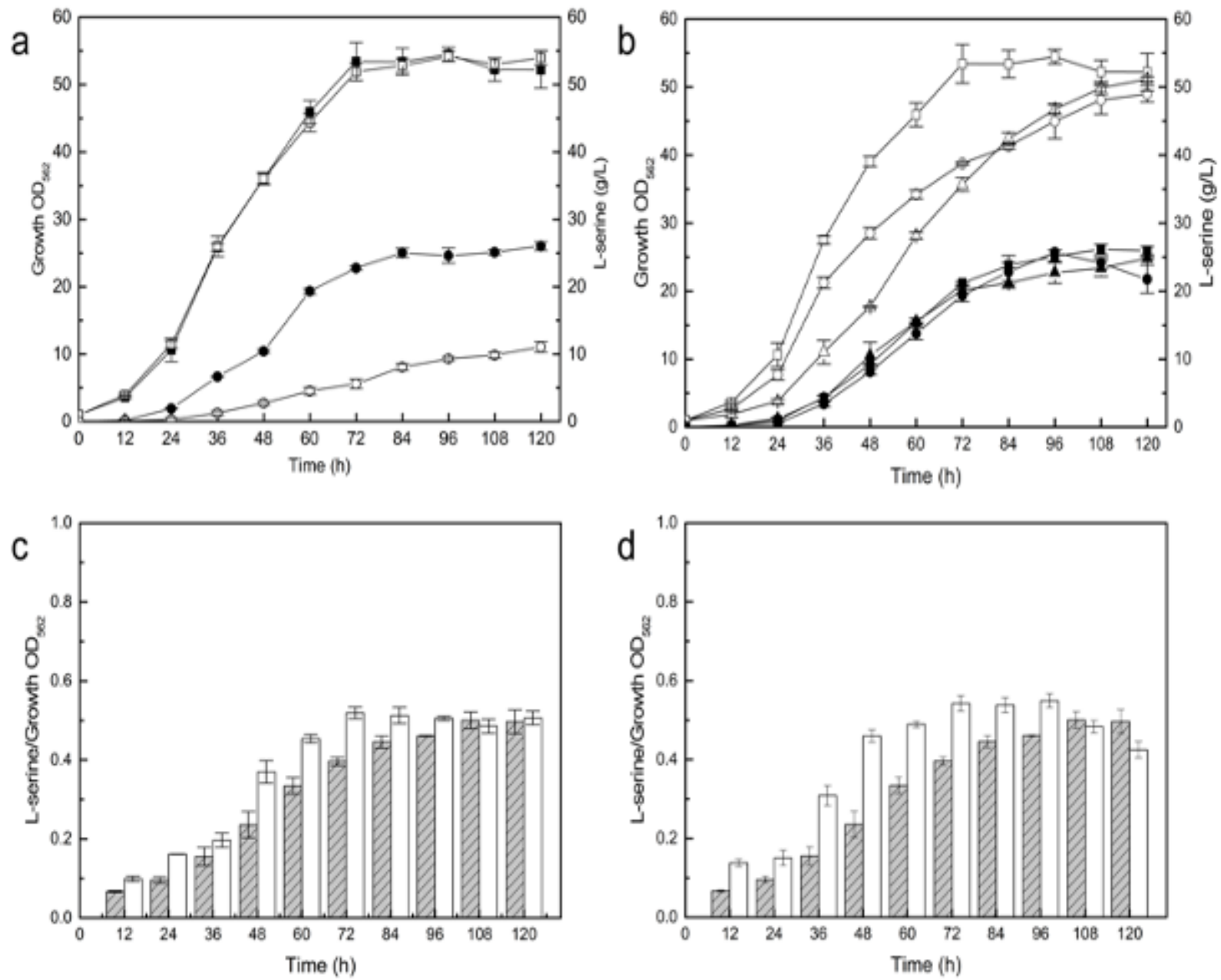

\section{Figure 4}

The effect of serE, NCgl0581 deletion or overexpression on SSAAI. (a) The cell growth (squares) and Lserine titer (circles) of SSAAI (solid symbols) and NCgl0581 deletion strain SSAAI $\triangle$ NCgl0581 (open symbols), respectively. (b) The cell growth (open symbols) and L-serine titer (solid symbols) of stain SSAAI (squares), NCgl0581 overexpression strain SSAAI-NCgI0581 (circles), NCgl0581 and serE double overexpression strain SSAAI-serE-NCgl0581 (triangles). (c) The yield of L-serine to biomass (Yp/x) of SSAAI (grey bar with slash) and NCgl0581 overexpression strain SSAAI-NCgl0581 (white bar). (d) The yield of L-serine to biomass ( $\mathrm{Yp} / \mathrm{x}$ ) of SSAAI (grey bar with slash) and NCgl0581 and serE double overexpression strain SSAAI-NCgl0581-serE (white bar). 


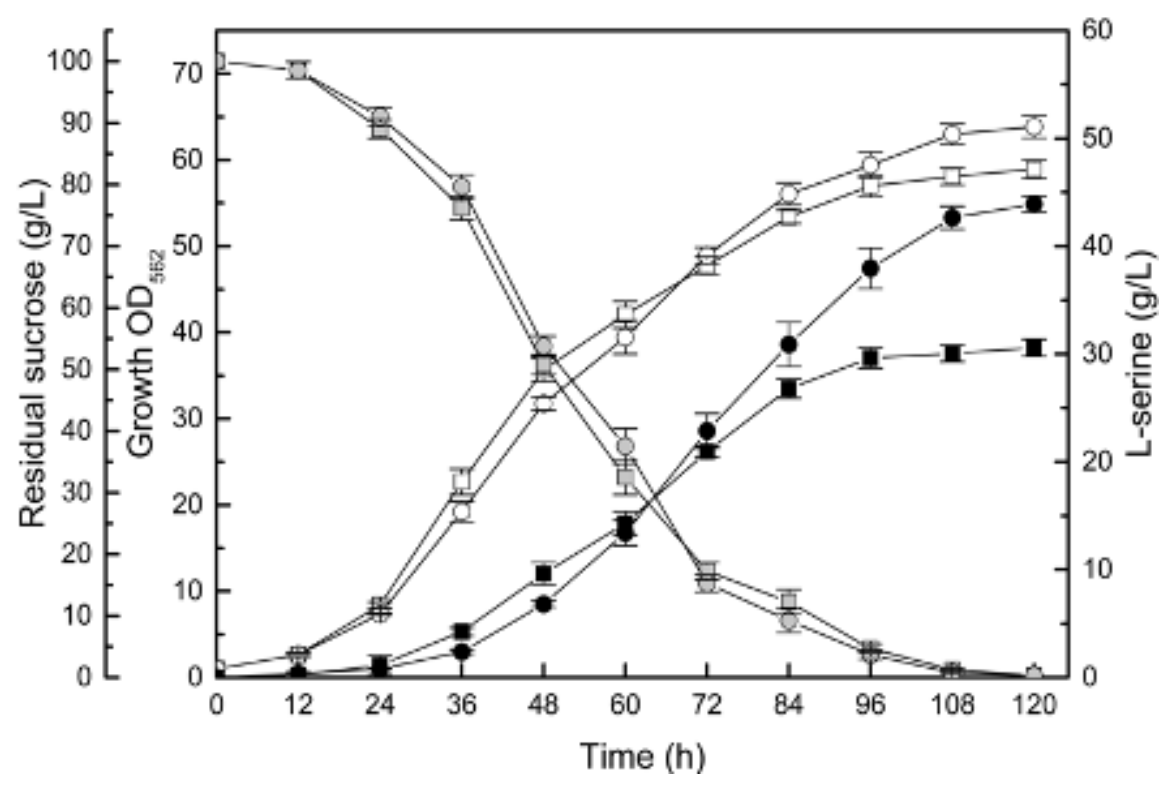

Figure 5

Fermentation process of strain A36 and strain A36-serA $\triangle 197$-serB-serC-serE.The cell growth (open symbols), L-serine titer (solid symbols) and residual sucrose (gray symbols) of stain A36 (squares) and A36-serA $\Delta 197$-serB-serC-serE (circles). Three parallel experiments were performed. Error bars indicate standard deviations of results from three parallel experiments.

\section{Supplementary Files}

This is a list of supplementary files associated with this preprint. Click to download.

- Supplementmaterials.docx 